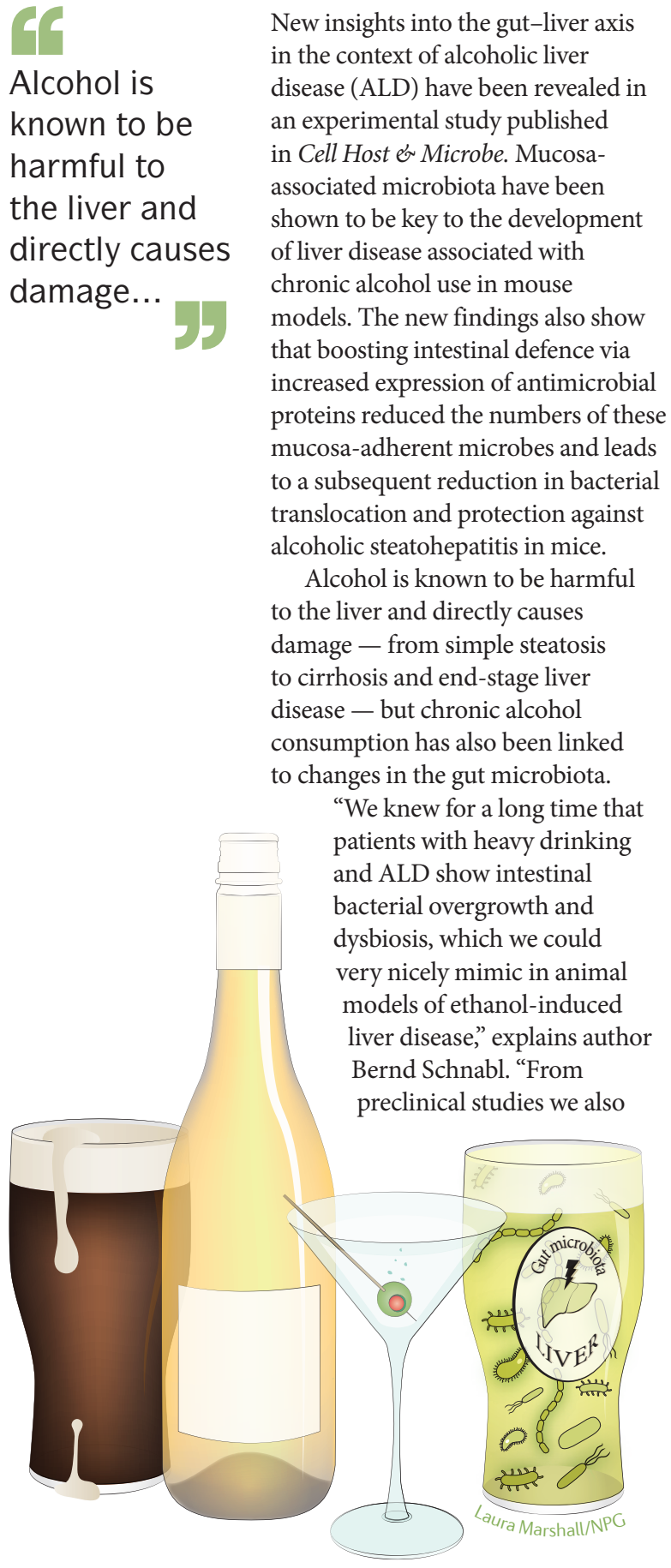

\title{
Mucosal microbes exacerbate experimental alcoholic steatohepatitis
}

knew that the gut microbiome is important, because non-absorbable antibiotics can prevent and treat alcoholic steatohepatitis," he adds, "but why changes in the intestinal microbiome occur was not known".

As such, the researchers wanted to explore the functional consequences of ALD. Previous work had already identified the association between low levels of the antimicrobial protein regenerating islet-derived protein 3 (REG3) and chronic alcoholic consumption, so the researchers focused their attention on these C-type lectins and whether absence or overexpression of these proteins affects the progression of ethanol-induced liver disease in mice.

The investigators showed that REG3B protected against ethanol-induced steatohepatitis without affecting intestinal absoprtion or hepatic metabolism of ethanol. In particular, Reg $3 b^{-/-}$ mice developed more severe ethanol-associated liver disease than wild-type littermate mice that were also subjected to chronic ethanol feeding. Furthermore, ethanol-fed Reg $3 b^{-/-}$mice had increased numbers of mucosa-associated bacteria compared with ethanol-fed wild-type mice; no difference in numbers and composition of luminal bacteria were observed between the mice. Moreover, this increased colonization of the intestinal surface was also matched by increased levels of bacterial translocation to the mesenteric lymph nodes and the liver. Similar results were observed with ethanol feeding in $R e g 3 g^{-1-}$ mice versus controls.

Importantly, intestinal overexpression of REG3G protected mice from ethanol-induced liver injury, including steatosis and inflammation, compared with controls. Interestingly, this overexpression also led to markedly reduced numbers of mucosa-associated bacteria and reduced bacterial translocation to mesenteric lymph nodes and the liver after ethanol feeding. Decreased numbers of luminal bacteria were also observed, as it was believed that overexpressed REG3G can penetrate the mucus layer into the intestinal lumen, mediating its antimicrobial effects there also. Bringing the findings back to the clinic, the researchers examined whole duodenal biopsy samples from patients with alcohol dependency and found that alcohol-dependent patients had increased numbers of adherent mucosa-associated microbiota in the small intestine compared with those without alcohol dependency.

"We now provide evidence that suppression of intestinal defence mechanisms leads to increased ALD by a mechanism that involves failure to control the adherent microbiota in the intestine," says Schnabl. Further work is planned to learn more about the underlying mechanisms to determine whether other molecules or strategies can increase REG3 expression, or indeed whether specific bacteria within the mucosa-associated microbiota exacerbate ALD. Targeting these microbes in particular might alter disease susceptibility.

Katrina Ray

ORIGINAL ARTICLE Wang, L. et al. Intestinal REG3 lectins protect against alcoholic steatohepatitis by reducing mucosa-associated microbiota and preventing bacterial translocation. Cell Host Microbe http://dx.doi.org/10.1016/ j.chom.2016.01.003 FURTHER READING Louvet, A. \& Mathurin, P. Alcoholic liver disease: mechanisms of injury and targeted treatment. Nat. Rev. Gastroenterol. Hepatol. 12, 231-242 (2015) 